Article

\title{
Determination of the Rate of Salt-Induced Rapid Coagulation of Polystyrene Latex Particles in Turbulent Flow Using Small Stirred Vessel
}

\author{
Oktaviani Oktaviani ${ }^{1,2}$ and Yasuhisa Adachi ${ }^{1, *}$ \\ 1 Graduate School of Life and Environmental Science, University of Tsukuba, 1-1-1 Tennodai, Tsukuba-shi, \\ Ibaraki 305-8572, Japan; okta_hanif@yahoo.com \\ 2 Center for Isotopes and Radiation Application, National Nuclear Energy Agency of Indonesia, \\ Jl. Lebak Bulus Raya No.49, Jakarta 12440, Indonesia \\ * Correspondence: adachi.yasuhisa.gu@u.tsukuba.ac.jp; Tel.: +81-298-534-862
}

Received: 10 November 2018; Accepted: 18 December 2018; Published: 24 December 2018

\begin{abstract}
In our study, we revisited a previously reported method for evaluating the mixing intensity of uniform colloidal spheres in terms of their collision frequency, with the aim of evaluating the validity of this method in the case of a small stirred vessel equipped with an impeller with four paddles. The rates of the salt-induced rapid coagulation of polystyrene latex (PSL) particles with five different diameters were measured as functions of the rotation rate. The ad-hoc assumption of the linear additivity of the perikinetics and the orthokinetics of the coagulation process was used for the analysis. Our previously proposed equation for the rate of turbulent coagulation as a function of the particle diameter, determined for an end-over-end rotation mixing device, was confirmed to be valid. However, it was found that, for small particles and low-mixing rates, i.e., for low Peclet numbers, the rate of coagulation becomes larger than that predicted on the basis of linear additivity because of the coupling effect of Brownian motion and the fluid flow during turbulent mixing. This increase occurred even though the rate was lowered by the wall effect, which resulted in an inhomogeneous distribution of the mixing intensity.
\end{abstract}

Keywords: collision frequency; polystyrene latex particle; coagulation rate; Peclet number; coupling effect; turbulent flow

\section{Introduction}

Coagulation is a phenomenon observed in many natural and engineered systems [1-5]. Most coagulation processes in practical applications occur under turbulent flow. Therefore, colloidal coagulation in turbulent flow can be regarded as one of the most fundamental and practically important issues in the domain of colloidal flocculation when one considers the engineering applications of the phenomenon. Accordingly, there have been numerous studies on this subject. A majority of these studies can be categorized into two groups. The first group is related to the prediction of the rate of coagulation in turbulent flow [6-9], while the other is related to the strength of the formed flocs against breakup under the stress of turbulent flow [10-12]. Since the length scale of interest in both cases is usually smaller than the Kolmogorov microscale of turbulence [13], most of the analyses involved have been performed on the basis of the concept of isotropic turbulence, which allow us to determine the statistical physical properties by the dimensional analysis using the rate of energy dissipation per unit mass and kinematic viscosity. Then, the mean value of velocity gradient can be derived. The dimension of this parameter is same as that of the shear rate.

The framework for the rate theory of coagulation was developed by Smoluchowski [14]. He derived the rate of coagulation in a laminar shear flow field while assuming that the colloid particles 
move along straight streaming lines until they undergo a collision. However, this motion is actually accompanied by perikinetics motion (i.e., Brownian motion). Using well-defined experiments involving the rapid coagulation of small latex particles, Swift and Friedlander [15] were able to confirm the validity of the predictions made on the basis of the Smoluchowski theory. They analyzed and experimentally determined the rate of coagulation based on the simple additivity of the perikinetics and orthokinetics motions (i.e., the motion determined by flow field) of the colloidal particles. In this case, the rate of collision by a reference particle $(J)$ can be written as

$$
J=\left(\alpha_{s} \frac{16}{3}+\alpha_{B} \frac{4 \pi}{P e}\right) 2 N(t) a_{0}^{3} \dot{\gamma}
$$

where the first and second terms represent the collision frequencies due to shear flow and Brownian motion, respectively. Further, $\alpha_{S}$ and $\alpha_{B}$ are the correction factors for the capture efficiency owing to the hydrodynamic interactions that occur upon the collision of the colloidal particles in the shear field and owing to Brownian motion, respectively. Finally, $N(t)$ denotes the total number of flocs per unit volume; $a_{0}$ is the radius of a singlet; and $P e$ is the transitional Peclet number, which is expressed as the ratio of the strength of the fluid flow and the diffusivity of the particles.

That is to say,

$$
P e=\frac{\dot{\gamma} a_{0}^{2}}{2 D}
$$

However, this simple treatment has been criticized by several researchers [16-18]. Based on a more rigorous treatment that takes into account the effect of the hydrodynamic interactions between two colloidal particles approaching each other along the trajectory determined through hydrodynamic analysis, Feke and Schowalter performed numerical modeling of the effect of Brownian motion on the rate of coagulation in a laminar shear flow. They reported that the numerically predicted values are considerably larger than those predicted based on the simple additivity for the $P e<50$ region. On the other hand, Van de Ven and Mason [18] investigated the effect of weak orthokinetic motion on perikinetic coagulation in the region corresponding to very small Peclet numbers, i.e., for $P e<1$, and concluded that there is no theoretical background for assuming that the principle of simple additivity is valid. It can be assumed that the effect of the coupling of Brownian motion and the fluid flow on the increase in the collision frequency is significant in the intermediate region $(1<P e<50)$. However, it should be noted that there exist neither numerical studies on shear coagulation nor experimental ones that confirm that the significant increase in the rate of coagulation in the intermediate region due to the coupling effect.

On the other hand, coagulation in turbulent flow is important from a practical viewpoint. Saffman and Turner [19] derived the rate theory for coagulation in turbulent flow on the basis of the isotropic nature of turbulent fluctuations. Higashitani et al. [6] further developed the theory by taking into account the effect of the hydrodynamic interactions, which essentially reduced the rate of coagulation. They also confirmed that turbulent flow can basically be treated as a type of shear flow. However, they did not determine the region corresponding to low $P e$ values, where a significant degree of coupling between Brownian motion and the fluid motion can be expected. In a previous work, Adachi et al. $[7,20,21]$ established a method for characterizing the turbulent flow in terms of the collision frequency between colloidal particles that is equivalent to the rate of coagulation during rapid coagulation. They performed measurements under a turbulent flow generated using an end-over-end mixer, wherein the average mixing intensity was mechanically fixed. A mixing flow was generated in a forked flask in which a very small amount of the colloidal dispersion $(10 \mathrm{~mL})$ was poured from one side of the forked flask to the other side. During this operation, the colloidal dispersion was mixed periodically by the rocking motion of the rotating device on which the forked flask was installed. During these experiments, $P e$ was varied by changing the diameter of the colloidal particles used. Although the control of the fluid motion seemed to be crude, their data confirmed the validity of the assumption of the simple additivity of the perikinetics and the orthokinetics. A minor point of concern 
with respect to their results was that a small quantitative discrepancy was observed in the absolute value of energy dissipation. That is to say, the rate of energy dissipation as calculated based on the rate of coagulation was smaller than that predicted from the loss of gravitational energy as estimated based on the mechanical configuration of the experimental setup used. The former was only $7 \%$ of the latter. Using a vessel equipped with a Rushton stirrer, Kobayashi and Adachi [22] also confirmed that the assumption of simple additivity is an appropriate one from a practical viewpoint. Their results also indicated that the rate of energy input as estimated from the measured torque is almost the same as that obtained from the rate of coagulation. It should be noted that they used a sufficiently large stirrer vessel for their experiments. However, in the case of small vessels, as will be discussed in the next section, the amount of energy consumed near the stirrer as well as that used near the wall and baffles of the vessel can be significantly large, leading to a reduction in the number of collisions. We term this effect "the wall effect." The wall effect becomes more pronounced with the increase in the thickness of the boundary layer in the case of low-mixing intensities. Therefore, one can assume the following scenario-the coupling effect enhances the rate of collision between the colloidal particles in the region corresponding to intermediate and low $P e$ values, while the wall effect reduces the rate of collision. In order to verify this hypothesis, we systematically measured the rate of coagulation for different mixing intensities using colloidal particles of different diameters in a small mixing stirrer vessel while focusing on intermediate and low $P e$ values. This setup is also relevant for studying bioreactors [23-25].

\section{Rate of Coagulation in Turbulent Flow}

\subsection{Rate of Coagulation during Turbulent Mixing}

The rate of collision of colloidal particles in turbulent flow is reflective of the characteristics of the flow. Therefore, the measured rate of coagulation can be regarded as an important parameter that describes the turbulent flow. Saffman and Turner [19] proposed a model for collisions between rain drops in clouds. They used the results of a statistical analysis of isotropic turbulence performed by Taylor [26], and assumed that the distribution of the velocity gradient, $\gamma$, can be approximated as a normal distribution [27] with a mean absolute value of

$$
|\bar{\gamma}|=\sqrt{\frac{4 \varepsilon}{15 \pi \nu}} .
$$

Further, they derived the rate of coagulation as follows:

$$
\frac{d N(t)}{d t}=-\alpha_{T} \sqrt{\frac{128 \pi \varepsilon}{15 v}} a_{0}^{3} N(t)^{2}
$$

where $\varepsilon$ is the rate of energy dissipation per unit mass and $v$ is the kinematic viscosity. Further, $\alpha_{T}$ denotes the correction factor for the collision efficiency and reflects the hydrodynamic interactions in turbulent flow. The significance of this factor with respect to collisions between colloidal particles immersed in a viscous fluid was highlighted by Higashitani [6]. The approximate value of this factor can be estimated using the numerical results reported by van de Ven and Mason [28], who determined its value for a simple shear field. By replacing the shear rate with its effective value in Equation (3), the value of the capture efficiency, $\alpha_{T}$, can be derived as shown below:

$$
\alpha_{T}=\left(\frac{A}{36 \pi \mu \sqrt{\frac{4 \varepsilon}{15 \pi v}} a_{0}^{3}}\right)^{k}
$$

where $A$ denotes the Hamaker constant and $\mu$ is the viscosity of the fluid in question. The reported values of $k$ for laminar flow are 0.18 for the case where there is no retardation effect of the Van der 
Waals force [28] and 0.25 when there is such an effect [29]. For the initial stage of coagulation, for which we can assume $N(t)^{2}=N(t) \times N(0)$, Equation (4) can be integrated as follows:

$$
\ln \frac{N(t)}{N(0)}=-\alpha_{T} \sqrt{\frac{128 \pi \varepsilon}{15 v}} N(0) a_{0}^{3} t
$$

Therefore, if we measure the rate of coagulation for the mono-dispersed colloidal particles using the known value of the Hamaker constant for rapid coagulation, we can estimate the value of $\varepsilon$, which characterizes the mixing process, from the rate of coagulation.

\subsection{Deviations in the Case of the System Corresponding to a Low-Mixing Intensity in a Small Vessel}

The above-mentioned rate equation is practically applicable for mixing with a sufficiently high Reynolds number. However, as noted in the Introduction section, the frequency of collisions between colloidal particles can be expected to be increased by the effect of Brownian motion. Feke and Schowalter showed both numerically and experimentally that the rate of coagulation in a laminar shear flow is enhanced by the effect of Brownian motion to a greater degree than that predicted on the basis of the simple addition of the contributions of Brownian motion and the fluid flow. Studies performed over the last two decades have numerically confirmed that a region exists wherein these is a considerable degree of coupling with Brownian motion, leading to an increase in the rate of collision that is higher than that expected based on the simple addition of the effects of the Brownian motion and the extensional fluid flow [30-32]. It has been suggested that the properties of the energy-dissipating structure of turbulent flow in the ultimate stage of the energy-transfer cascade can be approximated as a type of extension flow [33]. This is an important scenario based on the fact that the component of the strain tensor that contributes to energy dissipation is not rotational but extensional.

However, the experimental results of a previous study by Kobayashi and Adachi [22] confirmed the effectiveness of the simple additivity principle using a mixing vessel equipped with a Rushton-type stirrer. In order to explain these results, in this study, we introduced the concept of the heterogeneity of the turbulent flow. It is known that the value of $\varepsilon$ near the impeller or the wall of a vessel is much higher than that for the rest of the space. Let us consider this situation by introducing a scenario corresponding to two regions as illustrated in Figure 1. The region near the wall is termed the wall region and has a volume fraction of $\varnothing$ and rate of energy dissipation of $\varepsilon_{w}$. The rest of the space is termed as the bulk region and has a volume fraction of $(1-\varnothing)$ and rate of energy dissipation of $\varepsilon_{b}$. The average rate of energy dissipation can be written as

$$
\varepsilon_{a v}=(\varnothing) \varepsilon_{w}+(1-\varnothing) \varepsilon_{b}
$$

If we assume $\varnothing=0.1$ and $\varepsilon_{w}=10 \varepsilon_{b}$ and use the results of Equations (4) and (5) with $k=0.2$, the rate of coagulation based on $\varepsilon_{a v}$ can be expressed as

$$
\frac{d N(t)}{d t} \sim\left(1.9 \varepsilon_{b}\right)^{0.4} \sim 1.3\left(\varepsilon_{b}\right)^{0.4} .
$$

On the other hand, separate estimations for the wall and bulk regions result in

$$
\begin{gathered}
\left(\frac{d N}{d t}\right)_{w}+\left(\frac{d N}{d t}\right)_{b} \sim\left(0.1 \times 10 \varepsilon_{b}\right)^{0.4}+\left(0.9 \times \varepsilon_{b}\right)^{0.4}, \\
\left(\frac{d N}{d t}\right)_{w}+\left(\frac{d N}{d t}\right)_{b} \sim 2.0 \varepsilon^{0.4} .
\end{gathered}
$$

That is to say, the estimate made on the basis of the average value is smaller than that when separate calculations are performed. This deviation becomes larger as $\varnothing$ increases. In the case of a fully-developed turbulent flow, $\varnothing$ is expected to be small owing to the decrease in the thickness of 
the boundary layer. However, in the case of a weak turbulence, the effective value of $\varnothing$ will increase because of the effective increase in the thickness of the boundary layer. While we were not able to quantify the value of $\varnothing$, we could predict the reverse effect on the rate of coagulation for low-mixing intensities. Thus, the increase in the collision frequency due to the effect of the coupling of the fluid flow and Brownian motion can be considered to have been cancelled. This qualitatively explains the results of our previous study [22]. In order to confirm the presence of the coupling effect, more systematic measurements of the rate of coagulation for different mixing intensities and Peclet numbers are necessary. To this end, we measured the rate of rapid coagulation as a function of the mixing intensity using colloidal particles of different diameters.

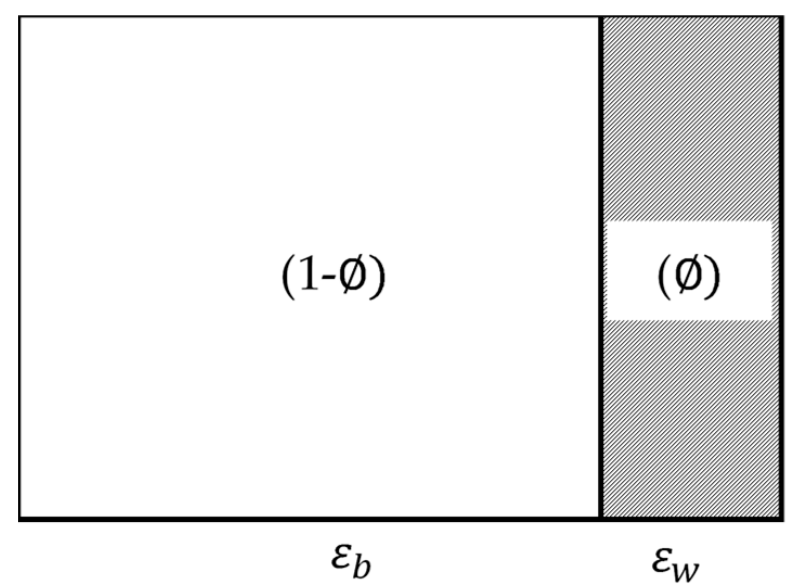

Figure 1. Schematic showing heterogeneity of $\varepsilon$ in a mixing vessel.

\section{Materials and Methods}

\subsection{Mixing Vessel Used}

The coagulation experiments were performed in a stirred vessel $(30 \mathrm{~mL}$, made by central workshop of Tsukuba University, Tsukuba, Japan) containing four baffles and equipped with a four-paddle impeller, as shown in Figure 2. Fluid motion is induced by the rotation of the impeller, which rotates at a constant angular velocity. If the kinetic energy provided by the rotating impeller is assumed to be balanced by the energy dissipated in the fluid, the value of $\varepsilon$ can be estimated from the following equation:

$$
\varepsilon=\varepsilon_{i}=\frac{2 \pi N_{r} T_{r}}{W}
$$

where $\varepsilon_{i}$ denotes the rate at which energy is input into the fluid per unit mass, $W$ is the mass of fluid in the vessel, $N_{r}$ is the rotational speed of the impeller, and $T_{r}$ is torque. Under these assumptions, $\varepsilon$ can be determined by measuring $T_{r}$ against $N_{r}$. For measuring $T_{r}$, a torque meter equipped with a rotary viscometer (Model LV, Brookfield) was employed. The obtained results are shown as a line in Figure 3. The data clearly confirm that $\varepsilon$ is proportional to the third power of $N_{r}$, as reported previously for mixers equipped with a paddle-type impeller. Further, the data obtained in the present study are found to keep with the extrapolated results for a Ruston-type turbine stirrer with a volume of $2.0 \times 10^{-3} \mathrm{~m}^{3}[22]$. 

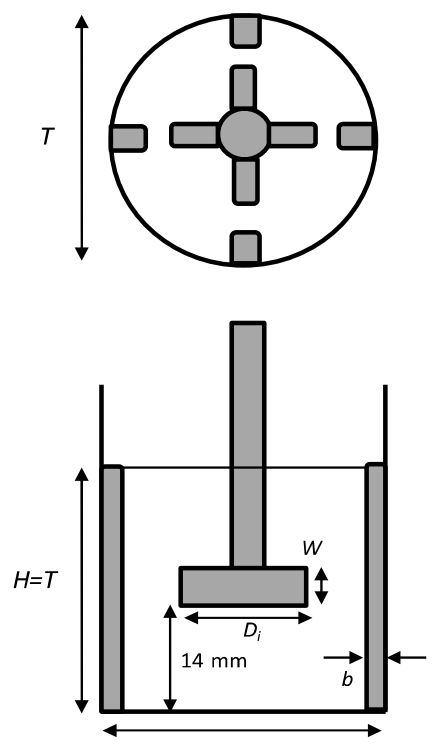

$T$

$H=T=35 \mathrm{~mm}, w=H / 5, b=H / 10, D_{i}=20 \mathrm{~mm}$

Figure 2. Mixing vessel and stirrer employed in the present study.

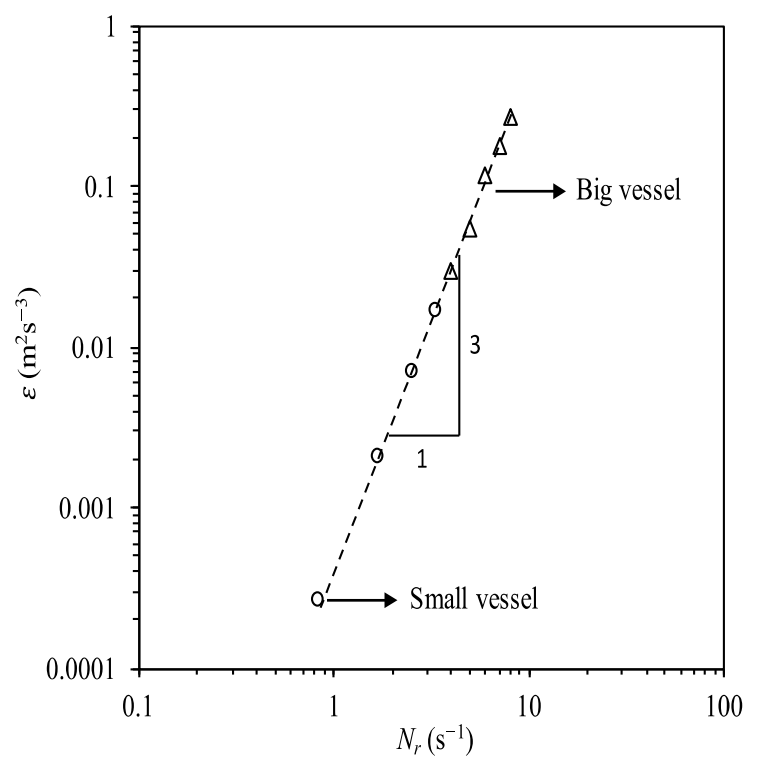

Figure 3. The value of $\varepsilon$ obtained using Equation (11). Its value was calculated based on the measured torque. A highly sensitive torque meter equipped with a rotary viscometer (Model LV, Brookfield) was used to measure the torque of the mixing device in Figure 2. Data for Rushton-type stirrer vessel with a volume of $2 \mathrm{~L}$ are plotted for reference [22].

\subsection{Materials Used}

Mono-dispersed polystyrene latex (PSL) spheres of five different diameters were prepared by the standard emulsion polymerization of styrene in the absence of a surfactant [34,35]. The PSL particle diameters and their number concentrations are listed in Table 1. The PSL suspension was sonicated for $20 \mathrm{~min}$ to eliminate any aggregate. In this procedure, an ultrasonic cleaner (AS ONE Co. model VS-100 $28 \mathrm{kHz} 100 \mathrm{~W})$ was employed. Potassium chloride $(\mathrm{KCl})$ was prepared and filtered through cellulose acetate filter paper prior to use. 
Table 1. Diameters and number concentrations of polystyrene latex particles.

\begin{tabular}{cc}
\hline Diameter $(\boldsymbol{\mu m})$ & Number Concentration $\times \mathbf{1 0}^{-\mathbf{7}}\left(\mathbf{c m}^{-\mathbf{3}}\right)$ \\
\hline 0.740 & $4.342 \pm 0.016$ \\
1.040 & $5.284 \pm 0.057$ \\
1.356 & $5.421 \pm 0.041$ \\
1.520 & $5.578 \pm 0.029$ \\
1.956 & $4.395 \pm 0.017$ \\
\hline
\end{tabular}

\subsection{Procedure}

To begin with, the vessel was filled with $15 \mathrm{~mL}$ of $\mathrm{KCl}$ solution. The impeller was set to rotate with a constant angular velocity, and $15 \mathrm{~mL}$ of the sonicated colloidal dispersion was poured into the vessel within approximately $1 \mathrm{~s}$. Figure 2 shows the mixing vessel used in this study. After a certain time, a small volume of the dispersion was sucked up, and $N(t)$ was measured as a function of the elapsed time using a Coulter counter (Beckman Coulter Inc., Brea, CA, USA).

\section{Results and Discussion}

\subsection{Evaluation of Rate of Coagulation}

The measured values of $\ln (N(t) / N(0))$ are plotted against $t \times N(0)$ for the different rotational speeds and particle diameters in Figure 4a-e. As can be seen from these figures, the relation between $\ln N(t)$ and $t$ was linear for each run. Assuming the simple additivity of the perikinetics and the orthokinetics, we can subtract the contribution of the Brownian flocculation from the slope of the relation between $\ln (N(t) / N(0))$ and $t$ while assuming its value to be $0.5 \times 4 k T / 3 \mu$ and obtain the rate of orthokinetic coagulation for each condition. The value of 0.5 was taken based on the results of a previous experimental study $[7,20]$. The fact that the relation was linear confirmed the suitability of Equation (6) for evaluating the effect of the turbulent mixing intensity on the coagulation rate. Further, the obtained results confirmed that a higher rotation speed causes more frequent collisions between the particles and hence increases the coagulation rate [6,22]. A comparison with the rate of coagulation in the case of an end-over-end mixing device $[7,20,21]$ indicated that the mixing intensity for the end-over-end rotation corresponds to the case with a rotation speed of $390 \mathrm{rpm}$ in terms of the frequency of collisions between the colloidal particles.

The obtained rates of orthokinetics coagulation are plotted as functions of the particle diameter in Figure 5. As can be seen from the figure, the plots of the coagulation rate against the particle diameter are parallel for higher mixing intensities. However, at lower mixing intensities, this relation deviates, suggesting a weaker dependence of the coagulation rate on the particle diameter and implying that the actual increase in the rate of coagulation is higher than that predicted based on the simple additivity for small particle diameters. This increase is reflective of the contribution of the coupling effect of Brownian motion and the shear flow. Further, this tendency can be seen more clearly in Figure 6, wherein the values of $\varepsilon$ obtained based on the rate of coagulation are plotted as functions of the rotation speed $\left(N_{r}\right)$ for different particle diameters. As can be seen from this figure, the rate of orthokinetic coagulation converges for higher $N_{r}$ values and increases for smaller $P e$ values, thus confirming the coupling effect. 
$N(0) t \times 10^{-15}\left(\mathrm{~m}^{-3} \mathrm{~s}\right)$

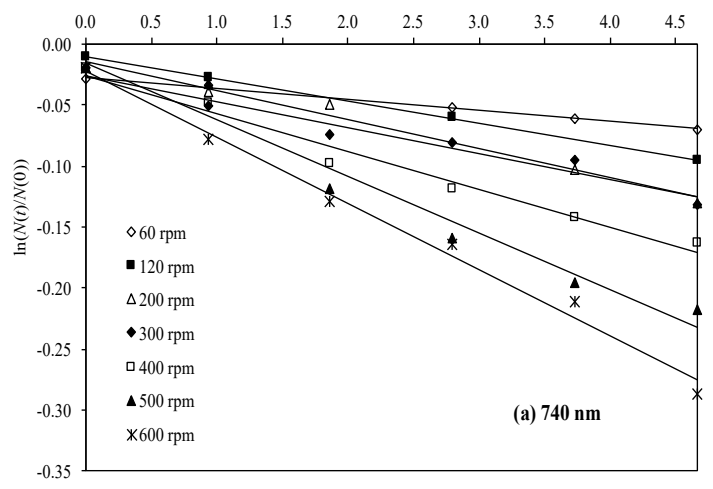

$N(0) t \times 10^{-15}\left(\mathrm{~m}^{-3} \mathrm{~s}\right)$

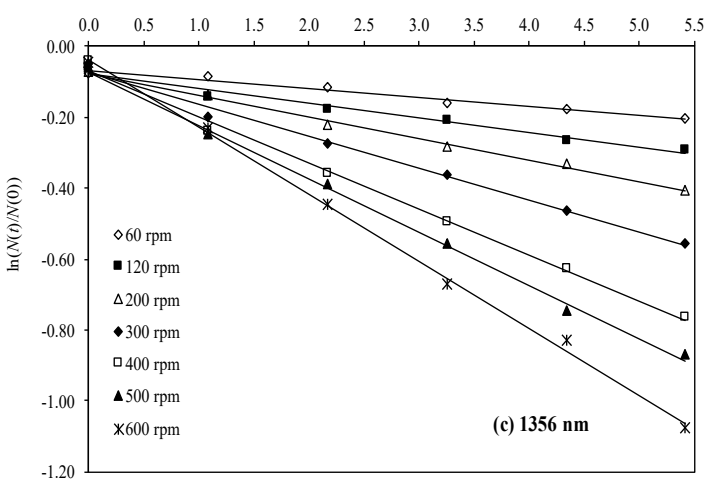

$N(0) t \times 10^{-15}\left(\mathrm{~m}^{-3} \mathrm{~s}\right)$

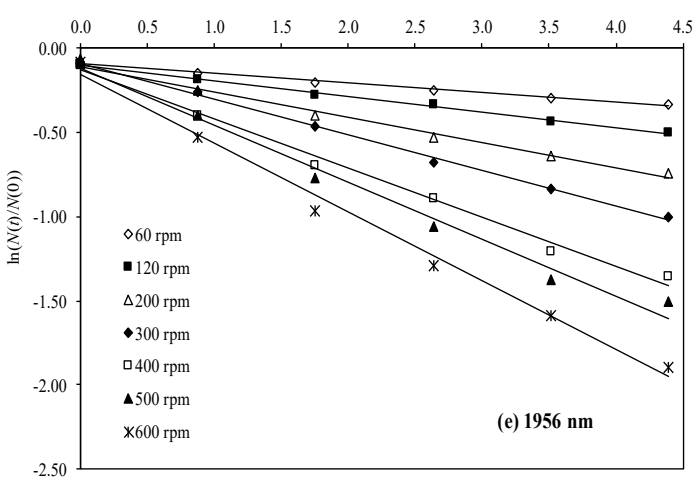

$N(0) t \times 10^{-15}\left(\mathrm{~m}^{-3} \mathrm{~s}\right)$

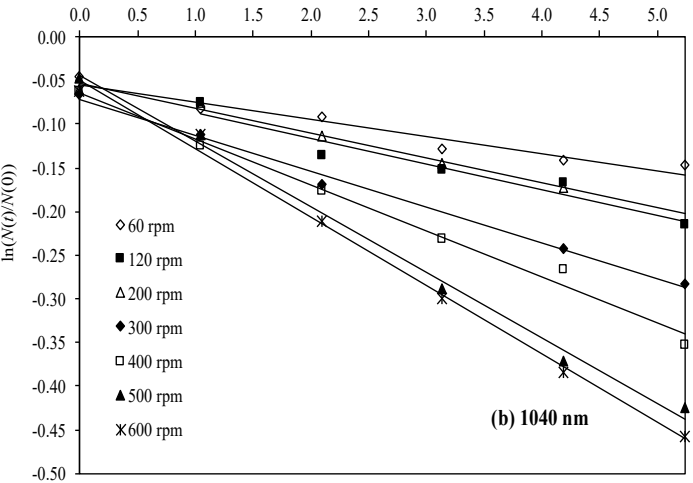

$N(0) t \times 10^{-15}\left(\mathrm{~m}^{-3} \mathrm{~s}\right)$

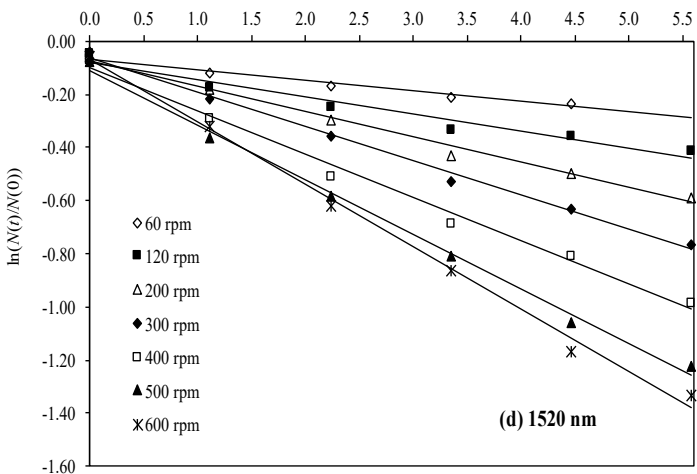

Figure 4. Temporal changes in the number concentration of colloidal particles of different diameters for different mixing intensities. 


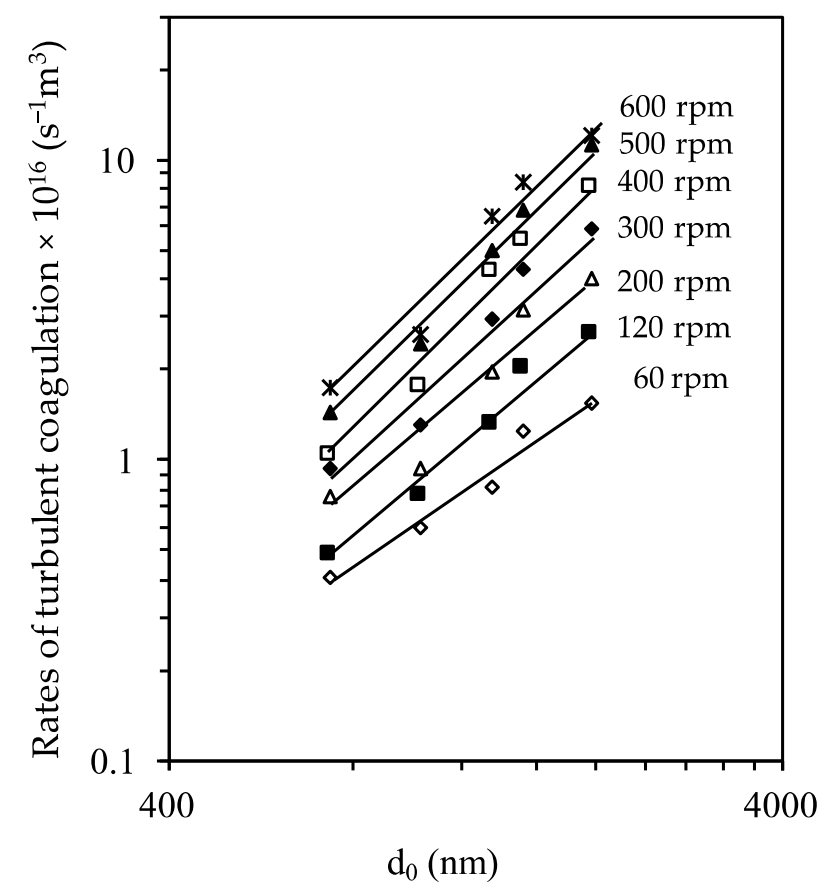

Figure 5. Rate of turbulent coagulation as a function of primary particle diameter for different rotation speeds $(60(\diamond), 120(\mathbf{\square}), 200(\triangle), 300(\diamond), 400(\square), 500(\Delta)$, and $600(*)[\mathrm{rpm}])$.

\subsection{Analyses of Wall and Coupling Effects for Low Peclet Numbers}

Assuming a value of $1.0 \times 10^{-21} \mathrm{~J}$ for the Hamaker constant, $\varepsilon$ was calculated from the data shown in Figure 5 using Equations (5) and (6). The calculation results are plotted in Figure 6 as functions of the rotation speed $\left(N_{r}\right)$. The calculated data principally lie between the solid and dashed lines. The solid line represents the values calculated from the measured torque using Equation (11). The result obtained in our previous experiment using latex particle of $1.0 \mu \mathrm{m}$ PSL particle and 2L mixing vessel equipped with four baffles and a Rushton-type impellor exactly corresponds to this line [22]. On the other hand, the dashed line is drawn assuming $7 \%$ of $\varepsilon_{i}$ is used for collision [7], which is the result of the rate of coagulation in the end-over-end mixing device $[7,35,36]$. In the low-mixing-intensity region, the curve for the plotted data bends upward. This tendency becomes more prominent for smaller particle diameters and reflects the contribution of the coupling effect, which enhances the rate of coagulation [16-18,20,30-32]. For higher mixing intensities, the plotted data curves are parallel to the curve for the energy input determined using the torque meter. The absolute value of the amount of energy dissipated per unit mass used for coagulation in the case of the higher mixing intensities is lower than the energy input, but higher than the energy dissipated per unit mass in the case of the end-over-end mixing device, which is represented by the dashed line. This result implies that, with a decrease in the diameter of the mixing vessel, the energy dissipated per unit mass, which is obtained from the rate of coagulation, will decrease. However, to confirm this trend, a more systematic study using mixing devices with similar geometries but different dimensions is required.

In order to elucidate the contribution of the coupling effect in the case of small Peclet numbers and the wall effect in the case of intermediate Peclet numbers, we replotted all the data for the rate of coagulation in Figure 5 as a function of the Peclet number in Figure 7. We theoretically determined the rate of coagulation as a function of the Peclet number, which is represented by the dashed line, using Equations (1) and (2). From the figure, one can easily recognize that for the region of higher Peclet number, c.a. $\mathrm{Pe}>50$, the slope of the plot converges to the theoretical value of $0.82(=1-0.18)$. However, for smaller Peclet number region, the rate of coagulation is higher than that predicted theoretically. The upward bending of the curve can be attributed to the contribution of the coupling effect, which enhances the coagulation rate. On the other hand, for higher Peclet numbers, the rate 
of coagulation decreased, owing to the wall effect. The wall effect ensures that the distribution of the dissipated energy is uneven in the small stirred vessel for low Reynold numbers [6,36-38]. Consequently, the rate of coagulation decreases owing to the wall effect. The same result was also obtained in a previous study [39]. However, when high Reynold numbers and a large stirred vessel were used by Kobayashi et al. [22], the wall effect was not detected. This is the reason they observed that the energy input and the energy used for coagulation are equal (i.e., $\varepsilon_{i}=\varepsilon_{d}$ ).

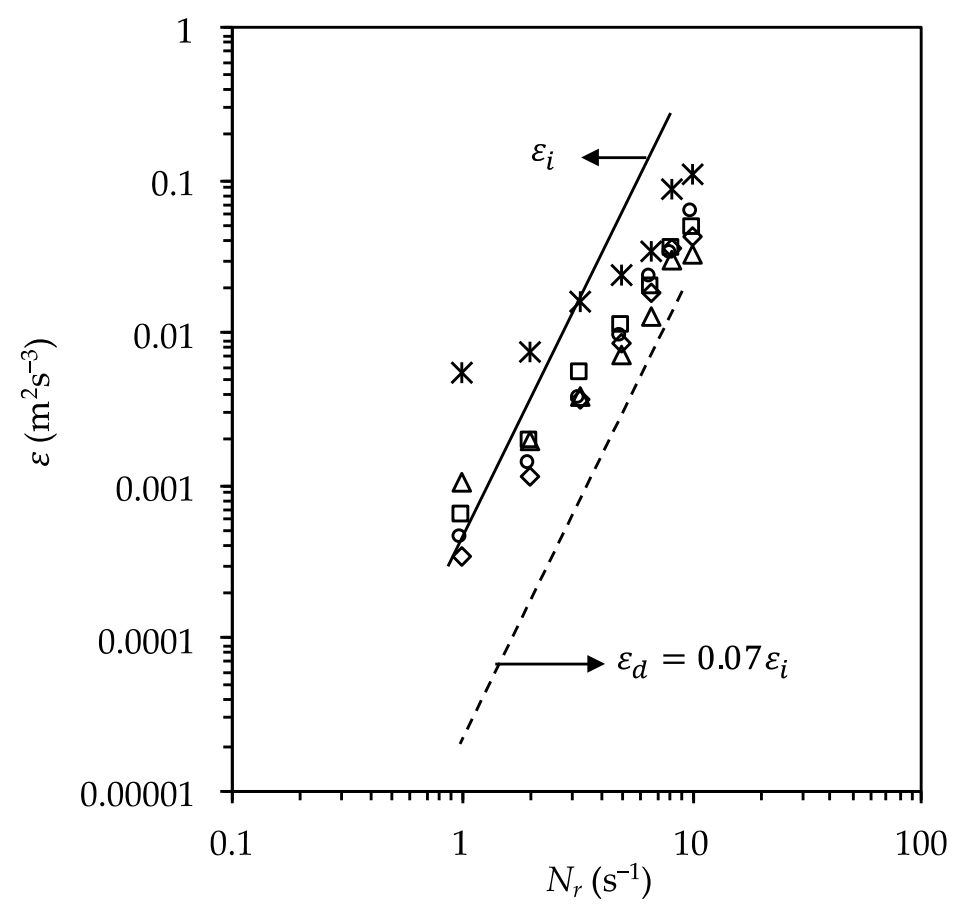

Figure 6. The values of $\varepsilon$ obtained using Equations (5) and (6) as the function of $N_{r}$ for different particle diameters $(740 \mathrm{~nm}(*), 1040 \mathrm{~nm}(\triangle), 1356 \mathrm{~nm}(\bigcirc), 1520 \mathrm{~nm}(\square)$, and $1956 \mathrm{~nm}(\diamond))$. Solid line $\left(\varepsilon_{i}\right)$ is the energy dissipated per unit mass obtained using the torque meter, while dashed line $\left(\varepsilon_{d}\right)$ is the energy used for coagulation in the case of an end-over-end mixing device as determined in a previous study [7].

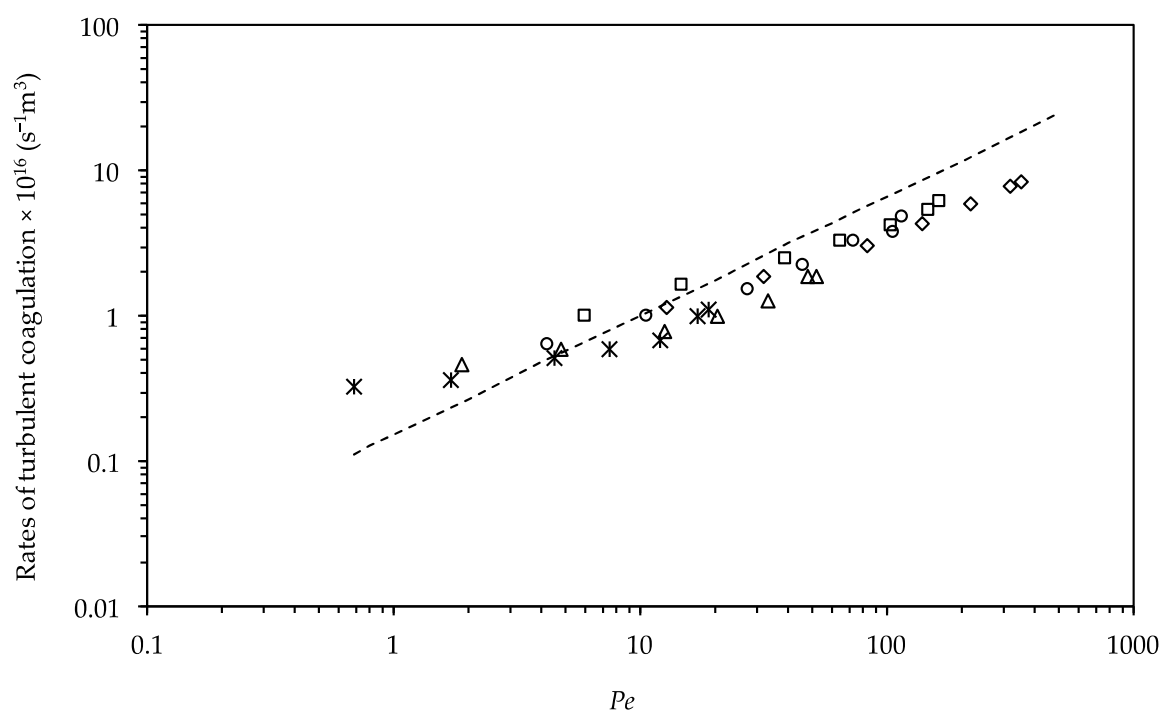

Figure 7. Data in Figure 5 replotted as a function of Peclet number. Symbols are the same as those in Figure 6 . The dashed line indicates theoretically predicted values. 
Currently, we are not able to quantify the contributions of the coupling and wall effects with respect to the rate of coagulation. However, we would like to mention the following three facts. Firstly, the upward bending of the curve for low Peclet numbers is indicative of the coupling effect, which increases the rate of coagulation. Secondly, the wall effect decreases the rate of coagulation. Finally, the amount of energy dissipated per unit mass depends on the size and shape of the mixing vessel used. Further studies are needed to quantitatively analyze the contributions of the wall and coupling effects with respect to shear coagulation, especially for low-mixing intensities. This should be done by using a systematical flow during the mixing process for which the Kolmogorov's microscale theory has a limitation on this region.

\section{Conclusions}

Mono-dispersed uniform spheres of PSL particles of five different diameters are coagulated in a small mixing vessel equipped with four baffles and a paddle-type stirrer. The relationship between $\ln (N(\mathrm{t}) / N(0))$ and $t \times N(0)$ was always linear, which confirmed that the turbulent mixing intensity can be evaluated in terms of the coagulation rate. A careful analysis into the additivity of the contribution of Brownian motion and turbulent flow on the rate of coagulation was performed to reveal the considerable effect of the coupling of Brownian motion and the shear flow for small Peclet number region. This result should be further investigated for other systems composed of much smaller particles, made up of different materials and with varying shapes. On the other hand, the idea of the wall effect was proposed to explain the effect of nonhomogeneous distribution of the energy dissipation on the rate of coagulation. The dissipation of the energy input near the wall of the small stirred vessel was not always negligibly small compared to that for the bulk region. Thus, it contributed to the lowering of the rate of coagulation. Finally, the amount of energy dissipated per unit mass was found to be significantly dependent of the size and shape of the mixing vessel used.

Author Contributions: Y.A. conceptualized the study, while O.O. performed the experiments. The original manuscript was prepared by O.O., while both O.O. and Y.A. had revised and finalized it. Y.A. supervised the entire study.

Funding: This work was funded by a Grant-in-Aid for Scientific Research (16H06382) from the Japanese Society of Promotion of Science. Oktaviani Oktaviani is thankful to the Ministry of Research, Technology, and Higher Education of Indonesia for a scholarship grant under the Research and Innovation in Science and Technology Project (RISET-PRO).

Acknowledgments: We thank Motoyoshi Kobayashi and Takuya Sugimoto for fruitful discussions. Central workshop of Tsukuba University is acknowledged for the fabrication of mixing vessel.

Conflicts of Interest: The authors declare no conflict of interest.

\section{References}

1. McAnally, W.H.; Mehta, A.J. Collisional Aggregation of Fine Estuarine Sediments. In Coastal and Estuarine Fine Sediment Processes; McAnally, W.H., Mehta, A.J., Eds.; Elsevier: Amsterdam, The Netherland, 2000; pp. 19-39.

2. Jackson, G.A. Coagulation Theory and Models of Oceanic Plankton Aggregation. In Flocculation in Natural and Engineered Environmental Systems; Droppo, I.G., Leppard, G.G., Liss, S.N., Milligan, T.G., Eds.; CRC Press: Boca Raton, FL, USA, 2005.

3. Farrow, J.B.; Swift, J.D. A new procedure for assessing the performance of flocculants. Int. J. Miner. Process. 1996, 46, 263-275. [CrossRef]

4. Rojas, O.J.; Hubbe, M.A. The dispersion science of papermaking. J. Dispers. Sci. Technol. 2005, 25, 713-732. [CrossRef]

5. Matilainen, A.; Vepsäläinen, M.; Sillanpää, M. Natural organic matter removal by coagulation during drinking water treatment: A review. Adv. Colloid Interface Sci. 2010, 159, 189-197. [CrossRef] [PubMed]

6. Higahshitani, K.; Yamauchi, Y.; Matsuno, Y.; Hosokawa, G. Turbulent coagulation of particles dispersed in a viscous fluid. J. Chem. Eng. Jpn. 1983, 16, 299-304. [CrossRef] 
7. Adachi, Y.; Cohen Stuart, M.A.; Fokkink, R. Kinetics of turbulent coagulation studied by means end-over-end rotation. J. Colloid Interface Sci. 1994, 165, 310-317. [CrossRef]

8. Kusters, K.A.; Wijers, J.G.; Thoenes, D. Aggregation kinetics of small particles in agitated vessel. Chem. Eng. Sci. 1997, 52, 107-121. [CrossRef]

9. Ehrl, L.; Soos, M.; Morbidelli, M.; Bäbler, M.U. Dependence of initial cluster aggregation kinetics on shear rate for particles of different sizes under turbulence. AIChE J. 2009, 55, 3076-3087. [CrossRef]

10. Kobayashi, M.; Adachi, Y.; Ooi, S. Breakup of fractal flocs in a turbulent flow. Langmuir 1999, 15, 4351-4356. [CrossRef]

11. Guérin, L.; Coufort-Saudejaud, C.; Liné, A.; Frances, C. Dynamics of aggregate size and shape properties under sequenced flocculation in a turbulent Taylor-Couette reactor. J. Colloid Interface Sci. 2017, 491, 167-178. [CrossRef]

12. Lachin, K.; Sauze, N.L.; Raimondi, N.D.M.; Aubin, J.; Gourdon, C.; Cabassud, M. Aggregation and breakup of acrylic latex particles inside millimetric scale reactors. Chem. Eng. Process. 2017, 113, 65-73. [CrossRef]

13. Kolmogorov, A.N. The local structure of turbulence in incompressible viscous fluid for very large Reynolds numbers. Dokl. Akad. Nauk SSSR 1941, 30, 301-305. [CrossRef]

14. Smoluchowski, M.V. Versuch einer mathematischen theorie der koagulationskinetik kolloider lösungen. Z. Phys. Chem. 1918, 92, 129-168. [CrossRef]

15. Swift, D.L.; Friedlander, S.K. The coagulation of hydrosols by Brownian motion and laminar shear flow. J. Colloid Sci. 1964, 19, 621-647. [CrossRef]

16. Feke, D.L.; Schowalter, W.R. The Effect of Brownian Diffusion on Shear-Induced Coagulation of Colloidal Dispersions. J. Fluid Mech. 1983, 133, 17-35. [CrossRef]

17. Feke, D.L.; Schowalter, W.R. The influence of Brownian diffusion on binary flow-induced collision rates in colloidal dispersions. J. Colloid Interface Sci. 1985, 106, 203-214. [CrossRef]

18. van de Ven, T.G.M.; Mason, S.G. The microrheology of colloidal dispersions VIII. Effect of shear on perikinetic doublet formation. Colloid Polym. Sci. 1977, 255, 794-804. [CrossRef]

19. Saffman, P.G.; Turner, J.S. On the Collision of Drops in Turbulent Clouds. J. Fluid Mech. 1956, 1, 16-30. [CrossRef]

20. Adachi, Y. Dynamic aspect of coagulation and flocculation. Adv. Colloid Interface Sci. 1995, 56, 1-31. [CrossRef]

21. Feng, L.; Stuart, M.C.; Adachi, Y. Dynamics of polyelectrolyte adsorption and colloidal flocculation upon mixing studied using mono-dispersed polystyrene latex particles. Adv. Colloid Interface Sci. 2015, 226, 101-114. [CrossRef]

22. Kobayashi, M.; Adachi, Y. Break-up Strength of Flocs Analyzed Using Orifice Converging Flow. Available online: https: / / ci.nii.ac.jp/naid/10029817970 (accessed on 17 December 2018).

23. Vallejos, J.R.; Kostov, Y.; Ram, A.; French, J.A.; Marten, M.R.; Rao, G. Optical analysis of liquid mixing in a minibioreactor. Biotechnol. Bioeng. 2006, 93, 906-911. [CrossRef] [PubMed]

24. Lamping, S.R.; Zhang, W.; Allen, B.; Shamlou, P.A. Design of a prototype miniature bioreactor for high throughput automated bioprocessing. Chem. Eng. Sci. 2003, 58, 747-758. [CrossRef]

25. Sullivan, D.M.; Lindsey, E.E. An approach to characterizing agitation by dispersion particle size. Ind. Eng. Chem. Fundam. 1962, 1, 87-93. [CrossRef]

26. Taylor, G.I. Statistical theory of turbulence. Proc. R. Soc. Lond. A 1935, 151, 421-444. [CrossRef]

27. Townsend, A.A. The Measurement of Double and Triple Correlation Derivatives in Isotropic Turbulence. Math. Proc. Camb. Philos. Soc. 1947, 43, 560-570. [CrossRef]

28. van de Ven, T.G.M.; Mason, S.G. The microrheology of colloidal dispersions VII. Orthokinetic doublet formation of spheres. Colloid Polym. Sci. 1977, 255, 468-479. [CrossRef]

29. Zeichner, G.R.; Schowalter, W.R. Use of trajectory analysis to study stability of colloidal dispersions in flow fields. AIChE J. 1977, 23, 243-254. [CrossRef]

30. Sheng-Hua, X.; Zhi-Wei, S.; Xu, L.; Wang, J.T. Coupling effect of Brownian motion and laminar shear flow on colloid coagulation: A Brownian dynamic simulation study. Chin. Phys. B 2012, 21, 1-8. [CrossRef]

31. Melis, S.; Verduyn, M.; Storti, G.; Morbidelli, M.; Bałdyga, J. Effect of fluid motion on the aggregation of small particles subject to interaction forces. AIChE J. 1999, 45, 1383-1393. [CrossRef]

32. Zinchenko, A.Z.; Davis, R.H. Collision rates of spherical drops or particles in a shear flow at arbitrary Peclet numbers. Phys. Fluids 1995, 7, 2310-2327. [CrossRef] 
33. Batchelor, G.K. Mass Transfer from Small Particles Suspended in Turbulent Fluid. J. Fluid Mech. 1980, 98, 609-623. [CrossRef]

34. Goodwin, J.W.; Hearn, J.; Ho, C.C.; Ottewill, R.H. Studies on the preparation and characterisation of monodisperse polystyrene lattice III. Preparation without added surface active agents. Colloid Polym. Sci. 1974, 252, 464-471. [CrossRef]

35. Aoki, K.; Adachi, Y. Kinetics of polyelectrolyte adsorption onto polystyrene latex particle studied using electrophoresis: Effects of molecular weight and ionic strength. J. Colloid Interface Sci. 2006, 300, 69-77. [CrossRef] [PubMed]

36. Cutter, L.A. Flow and turbulence in a stirred tank. AIChE J. 1966, 12, 35-45. [CrossRef]

37. $\mathrm{Ng}, \mathrm{K}$.; Yianneskis, M. Observations on the distribution of energy dissipation in stirred vessels. Trans IChemE A. 2000, 78, 334-341. [CrossRef]

38. Zhou, G.; Kresta, S.M. Distribution of energy between convective and turbulent flow for three frequently used impellers. Trans IChemE A. 1996, 74, 379-389.

39. Oktaviani, O.; Adachi, Y. Effect of mixing intensity on flocculation kinetics of polystyrene latex particles with high-charge density polyelectrolyte at various ionic strength. Colloid Polym. Sci. 2018, 296, 1945-1951. [CrossRef]

(C) 2018 by the authors. Licensee MDPI, Basel, Switzerland. This article is an open access article distributed under the terms and conditions of the Creative Commons Attribution (CC BY) license (http://creativecommons.org/licenses/by/4.0/). 